\title{
A convolutional neural network aided physical model improvement for AC solenoid valves diagnosis
}

\author{
Georges Tod* \\ CoDesignS \\ Flanders Make \\ Leuven, Belgium \\ georges.tod@flandersmake.be
}

\author{
Agusmian Partogi Ompusunggu \\ DecisionS \\ Flanders Make \\ Leuven, Belgium \\ agusmian.ompusunggu@flandersmake.be
}

\author{
Tamir Mazaev \\ IDLab \\ Ghent University \\ Ghent, Belgium \\ tamir.mazaev@ugent.be
}

\author{
Erik Hostens \\ DecisionS \\ Flanders Make \\ Leuven, Belgium \\ erik.hostens@flandersmake.be
}

\author{
Kerem Eryilmaz \\ DecisionS \\ Flanders Make \\ Leuven, Belgium \\ kerem.eryilmaz@flandersmake.be
}

\author{
Sofie Van Hoecke \\ IDLab \\ Ghent University \\ Ghent, Belgium \\ sofie.vanhoecke@ugent.be
}

\begin{abstract}
This paper focuses on the development of a physics-based diagnostic tool for alternating current (AC) solenoid valves which are categorized as critical components of many machines used in the process industry. Signal processing and machine learning based approaches have been proposed in the literature to diagnose the health state of solenoid valves. However, the approaches do not give a physical explanation of the failure modes. In this work, being capable of diagnosing failure modes while using a physically interpretable model is proposed. Feature attribution methods are applied to CNN on a large data set of the current signals acquired from accelerated life tests of several AC solenoid valves. The results reveal important regions of interest on current signals that guide the modeling of the main missing component of an existing physical model. Two model parameters, which are the shading ring and kinetic coulomb forces, are then identified using current measurements along the lifetime of valves. Consistent trends are found for both parameters allowing to diagnose the failure modes of the solenoid valves. Future work will consist of not only diagnosing the failure modes, but also of predicting the remaining useful life.
\end{abstract}

Keywords-alternating current solenoid valve, convolutional neural network, feature attribution methods, shading ring force, condition monitoring

\section{NOMENCLATURE}

$\begin{array}{cl}\phi(t) & \text { Magnetic flux } \\ N & \text { Number of main coil windings } \\ V(t) & \text { Input voltage } \\ i(t) & \text { Output current } \\ R & \text { Coil resistance } \\ m & \text { Mass of the plunger } \\ c & \text { Damping coefficient } \\ k & \text { Return spring stiffness } \\ F_{p} & \text { Return spring pretension force } \\ F_{s} & \text { Force applied by the solenoid on the plunger } \\ m m f & \text { Magnetomotive force } \\ H_{k} & \text { Magnetic field intensity through } k \\ l_{k} & \text { Length of } k \\ \mu_{0} & \text { Air permeability } \\ A_{x x} & \text { Cross-sectional area of } x x\end{array}$

978-1-7281-0329-7/19/\$31.00 (C2019 European Union

\section{INTRODUCTION}

Fault diagnosis of critical components of a production machine is paramount importance to avoid the machine breakdowns that can lead to production losses. Solenoid valves are critical components of many machines and used in many industrial applications, like heavy duty gas turbine engines, to regulate the fluid and gas flow. Therefore, condition monitoring and diagnosis of solenoid valves plays an important role in many industrial applications. Signal processing [1] and machine learning based approaches [2] have been proposed in the literature to diagnose the state of solenoid valves resulting in the development of a sensor to detect anomalies [3] or a method to cluster failures [2]. None of these approaches give a physical explanation of the failure modes nor their end of useful life. The physical models of valves are derived from first principles allow to predict the current signal in a valve [4,5] or qualitatively explain current signals [1]. In this work, being capable of diagnosing failure modes while using a physically interpretable model is proposed. To reach our goal, machine learning techniques applied to a large data set of current measurements (section II) reveal important regions in current signals (section III). This way, the highlighted regions in the current signals have guided us to identify the main missing components from the existing physical models [4,5] of AC solenoid valves (section IV). Expertise is applied to update the physical model with two additional forces, namely shading and kinetic Coulomb friction forces. The forces are then identified using the current signal measurements along the lifetime of valves. The identification results show consistent trends observed for both forces allowing to diagnose the failure modes of the solenoid valves. Therefore, the new physical model proposed in this paper can be used to diagnose AC solenoid valves. 


\section{EXPERIMENTAL MEASUREMENT CAMPAIGN}

A setup was built in order to age valves from new to their end of useful life. The solenoid valves that were tested are direct acting 3/2 way normally closed solenoid valves. The endurance test consisted of 12000 acquisitions per valve of 10 on-off switches $(\sim 4 s)$ performed every 3 minutes. Each valve was supplied with an input voltage of $110 \mathrm{~V} \mathrm{AC}$ at $50 \mathrm{~Hz}$. For every test, the currents were measured. Piezo patches were placed on the top of the valves to measure when the plunger would hit the stopper, see figure 1.

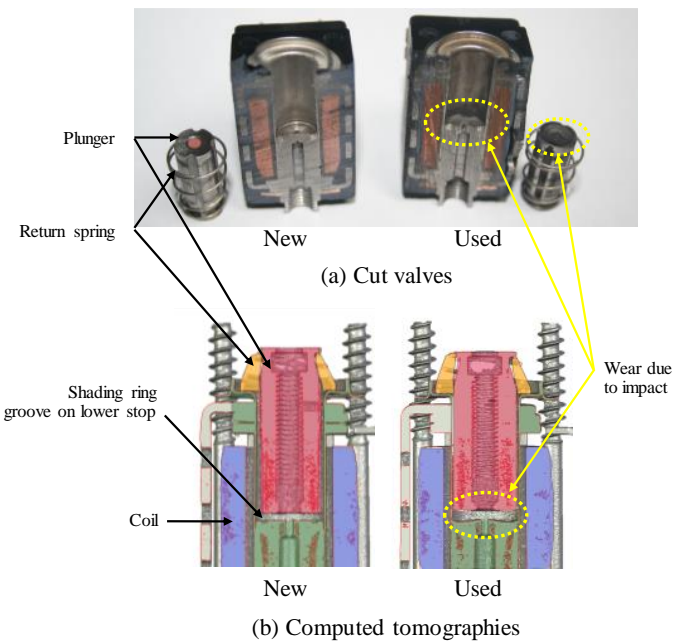

Fig. 1. New solenoid valve (left) vs. used one (right). During valve opening, plunger impacts lower stop.

The behavior of the valves could be classified into three distinct classes, based on the measurements of the accelerometer signals. A sample of each class is shown in Figure 2. At the beginning of its lifetime, it is observed that closing the valve results in one isolated hit of the plunger. This is the expected behavior of a healthy valve (class 0 ). After this healthy period, a second regime is observed in the accelerometer signal where multiple hits are visible (class 1). The last regime is when no hits are observed in the vibration signal anymore (class 2). During its lifetime, it is observed that a valve sporadically goes back from class 1 to class 0 . However, once the valve shows no hits anymore, it consistently remains in this regime.

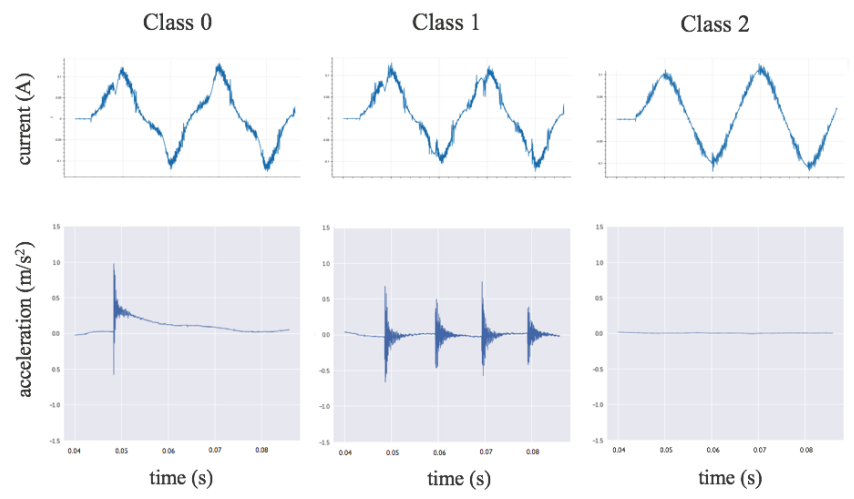

Fig. 2. Samples of the current and accelerometer signals given per class

\section{FINDING THE UNMODELLED COMPONENT USING MACHINE LEARNING}

\section{A. Regions of importance from current signals}

At the start of this study, the physical origin of the anomalous plunger behavior was unknown. In order to gain insight into this problem, we formulate the classification of the three classes as a supervised machine learning task. Each current signal is labelled based on its corresponding accelerometer signal, which the learning algorithm should be able to predict based on the current signal alone. Subsequently, the prediction model of the classifier is analyzed. An overview of the size and dimensionality of the dataset is given in Table I. Note that the number of instances for all three classes is balanced, which means that the classification accuracy is an appropriate metric for evaluation. All current signals are normalized to a zero mean and a standard deviation of one (calculated from the training set).

TABLE I SPECIFICATIONS OF THE SOLENOID VALVE DATA FOR THE CLASSIFICATION TASK.

\begin{tabular}{|l|l|}
\hline Description & Value \\
\hline Total \# of valves & 16 \\
\hline \# of time steps in current sample & 2300 \\
\hline \# of valves in training set & 12 \\
\hline \# of valves in test set & 4 \\
\hline \# of classes & 3 \\
\hline \# of samples per class, per valve & 20 \\
\hline Total \# of training samples & 720 \\
\hline Total \# of test samples & 240 \\
\hline
\end{tabular}

We compare the performance of 3 different classification algorithms on the raw current signal:

1) A support vector machine (SVM) using a radial basis function $(\mathrm{RBF})$ kernel [6].

2) A multilayer perceptron (MLP) with two hidden layers containing 64 neurons each [7].

3) A convolutional neural network $(\mathrm{CNN})$ architecture as designed by Ince et al. [8].

The hyperparameters of the SVM are set using a grid search with stratified cross-validation on 5 partitions of the training set with a validation set of $20 \%$ per partition. Both the MLP and the CNN are trained with the Adam algorithm, optimized on a categorical cross-entropy loss. A dropout rate of 0.5 is applied to the hidden layers of the MLP in order to prevent overfitting. Every classifier is also evaluated ten times on a differently chosen division of 12 training and 4 test valves.

We employ the CNN architecture of Ince, et al., since the classification task for which it was designed is very similar to our use case in terms of the dimensionality of the dataset and its application domain (motor fault detection based on raw current signals). Using a deep architecture such as a CNN prevents the need for engineering features from raw sensor data. MLPs and SVMs are commonly utilized as baselines to which a deep learning approach for fault diagnosis is compared to [9]. 
In order to gain insight into the predictions of the $\mathrm{CNN}$, we apply different feature attribution methods (Occlusion, DeepLIFT, Layer-wise Relevance Propagation, Integrated Gradients, Gradient*Input). These methods aim to highlight regions in the input signal of the neural network which influence the prediction model the most. Details on the heuristics used by these methods to assign importance to the input can be found in [10]. In this work, the benefit of these insights is that they can be leveraged to update a physical model of the valves. This is the topic of discussion in Section III.

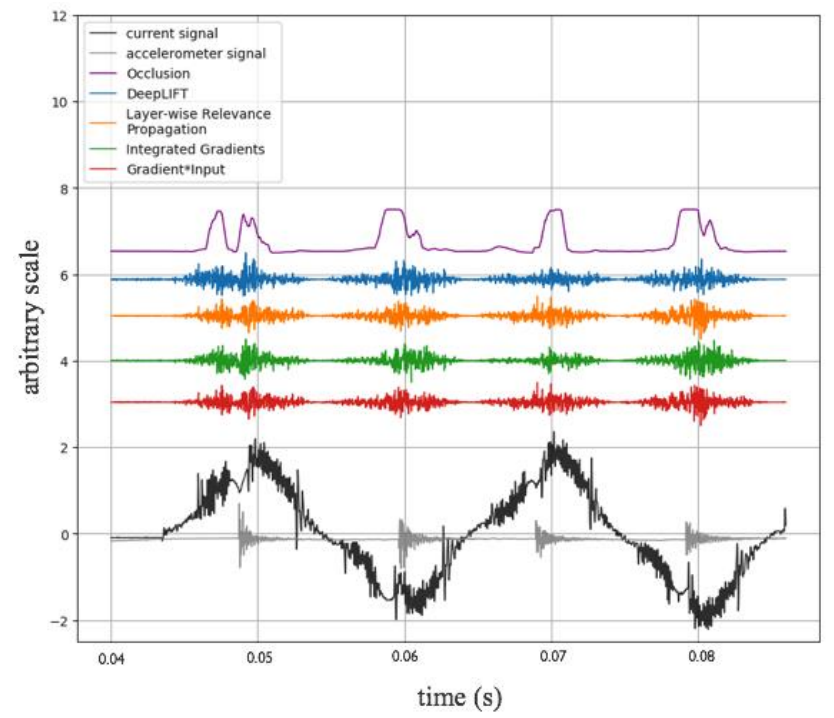

Fig. 3. Important regions of a current signal as returned by different feature attributions methods applied to a $\mathrm{CNN}$ trained on the classification task. The attribution values are scaled in amplitude and given an offset for visibility.

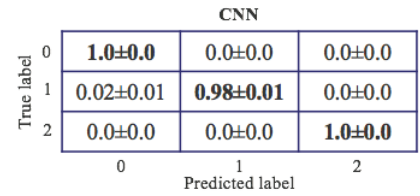

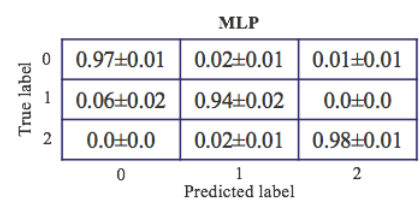

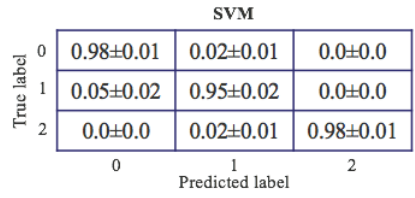

Fig. 4. Normalized confusion matrices of the test set performance for 3 different classifiers. The mean and standard deviation are given for 10 evaluations with a differently chosen set of 4 test valves per evaluation.

\section{B. Numerical results}

The normalized confusion matrices for the test set performance of the three classifiers are given in Figure 3. The best result for all three classes is obtained with the $\mathrm{CNN}$, which means it has the best accuracy overall $(0.99$, compared 0.96 for the MLP and 0.97 for the SVM). In Figure 4 one example of a current sample of class 0 is shown, together with the output of different feature attribution methods applied to the CNN trained on the classification task. It shows that CNN mainly assigns importance to 4 distinct regions of the signal when it classifies this current signal. This is observed for all samples in the dataset. The physical relevance of these regions will be described in detail in the next section.

\section{UPDATING THE PHYSICAL MODEL}

\section{A. Missing forces in healthy valve models}

Solenoid valve physical models in literature [1,4,5] are electro-magneto-mechanical models that can be represented in their simplest form using the first equation for the magnetic flux $(\phi)$ and a second one for the plunger motion, see equations (1). These models can correctly explain the shape of the current in healthy conditions (see class 0 from figure 2). However, they cannot explain unhealthy conditions (see classes $1 \& 2$ from figure 2), revealing something remains unmodelled. The input of such a system is a sinusoidal voltage $(V)$ applied to the valve and experimentally only the current $(i)$ is measured. It is difficult to measure the plunger motion, but the accelerometer placed at the top of the valves still allows one to measure plunger hits on the stops, revealing its position instantaneously.

$$
\left\{\begin{array}{c}
\dot{\phi}=\frac{1}{N}(V-R i) \\
\dot{x}=\frac{1}{m}\left(-c \dot{x}-k x-F_{p}+F_{s}\right)
\end{array}\right.
$$

The magnetic flux and the plunger motion equations are coupled through the plunger position $(x)$. Its position changes the magnetomotive force $(\mathrm{mmf})$ by varying the ratio air/iron the magnetic flux must cross (mainly) axially, see equations (2-4).

$$
\begin{aligned}
& \mathrm{i}=\frac{m m f}{N}=\frac{l}{N} \sum_{\mathrm{k}} H_{k} \cdot l_{k} \\
& H_{\text {air } \_x}=\frac{\phi}{\mu_{0} \cdot A_{x x}} \text { with } x x=\{\text { radial, axial }\} \\
& H_{y y}=H_{\text {iron }}\left(\frac{\phi}{A_{y y}}\right) \text { with } y y=\{\text { housing,plunger }\}
\end{aligned}
$$

As explained in the previous paragraph, feature attribution methods revealed that important regions of the current signal can be found around current extrema, see figure 4. Additionally, around these regions and when the valves are degraded, accelerometers measured plunger impacts, see class 1 degradation from figure 2 and $5 \mathrm{~b}$. Physically, this could be interpreted as the return spring, see figure 1, winning temporarily the force balance contest provoking a downward movement of the plunger. When the plunger moves down 
temporarily, the sealing of the valve will be temporarily

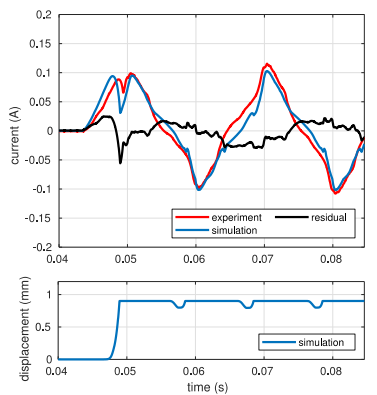

(a)

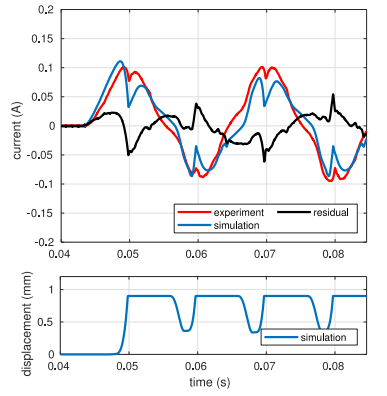

(b)

compromised, and the valve will leak.

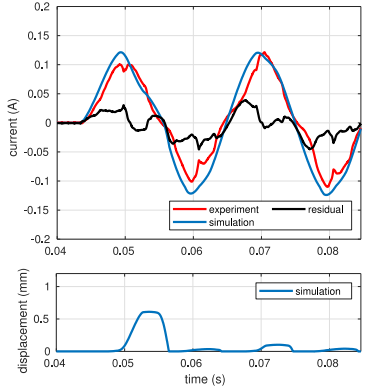

(c)

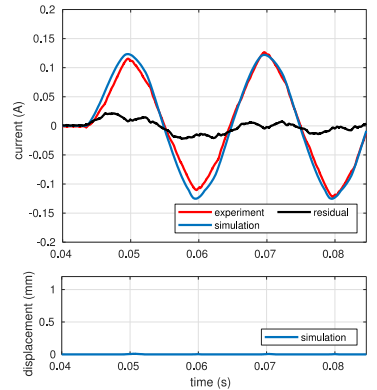

(d)

Fig. 5. Current signal signatures: (a) healthy valve, (b) leakage (large plunger motion), (c) erratic plunger motion and (d) plunger is stuck

As soon as the current is high enough again, the electromagnetic force applied by the coil on the plunger becomes high enough to bring the plunger back to the stop and recover the sealing. As this temporary loss of force counter acting the return spring is not seen in healthy conditions; the assumption can be made that a force pulling the plunger up in healthy conditions disappears after degradation.

$$
\left\{\begin{array}{c}
\dot{\phi}=\frac{1}{N}(V-R i) \\
\dot{x}=\frac{1}{m}\left(-c \dot{x}-k x-F_{p}+F_{s}+F_{c}+F_{s r}\right)
\end{array}\right.
$$

Around the region where the unmodelled force plays an important role, the current just crossed zero, meaning the electromagnetic force applied by the main coil on the plunger is null. To avoid the counteracting force on the return spring to be null, valve manufacturers use a secondary source of electromagnetic force from a shading ring, see figure 1 . The coil magnetic field induces an electric current in the shading ring that produces an induced electromagnetic force that delayed of $1 / 4$ of a cycle from the coil current allows to avoid a null electromagnetic force when the current is null. This additional force $\left(F_{s r}\right)$ is introduced to the initial model, see equations (5). In this work, it is proposed to simply model the shading ring force as a periodic function delayed from the main coil force, see figure 6 . The fact that this force might decrease during the lifetime of a valve is an assumption that is thought to be acceptable based on the results from the computed tomographies where it can be clearly seen that the zone of impact of the plunger is the zone where valve manufacturers place the shading ring (see figure 1). More confidence in this assumption is also gained by the numerical results of the next paragraph, see figure 9. Finally, visual and manual actuation of damaged valves revealed the need to add friction, modeled as a Coulomb friction force ( $F_{c}$, containing both static and dynamic terms), see equation (5).

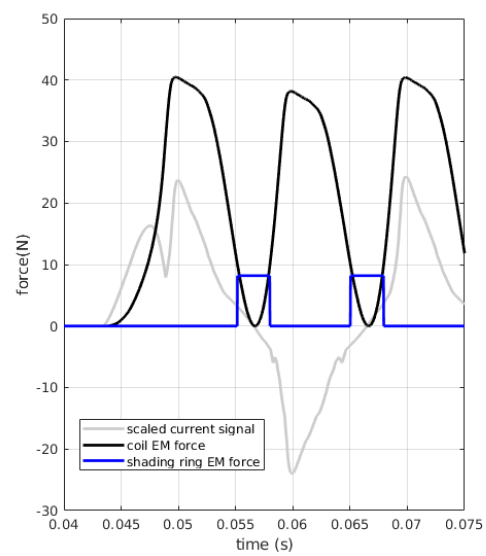

Fig. 6. Additional shading ring force
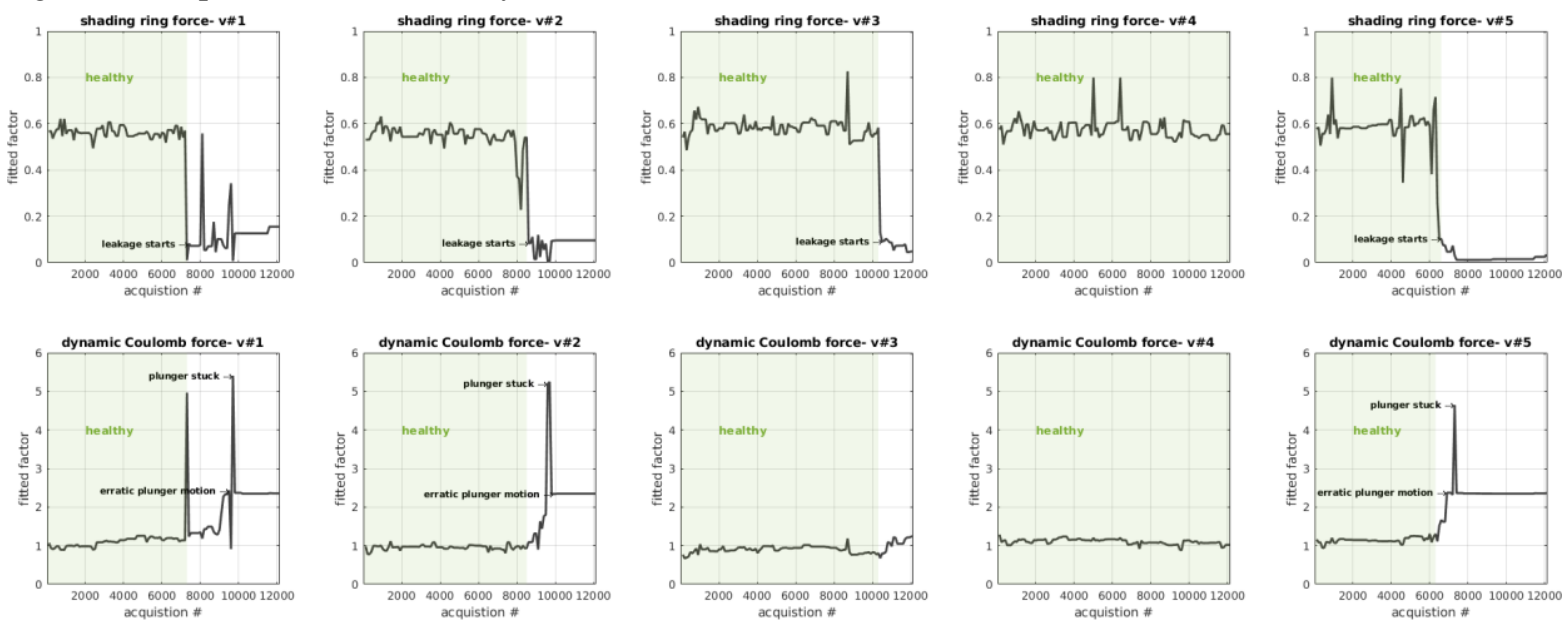

Fig. 7. Model-based solenoid valve condition monitoring. Two parameters (shading ring and dynamic Coulomb friction force) are identified on experimental data using the proposed physics driven model. 


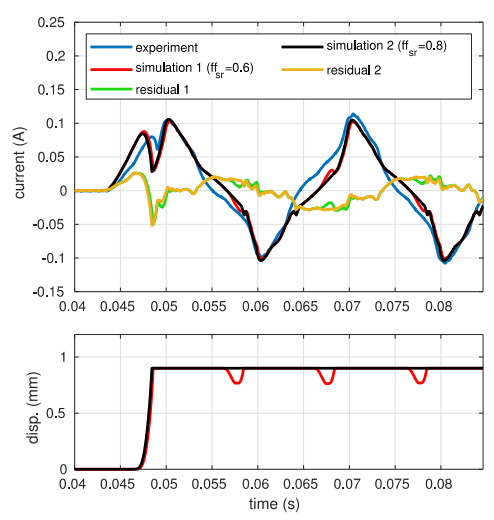

Fig. 8. Current and displacement signatures for varying shading ring forces fitting factors (ffsr)

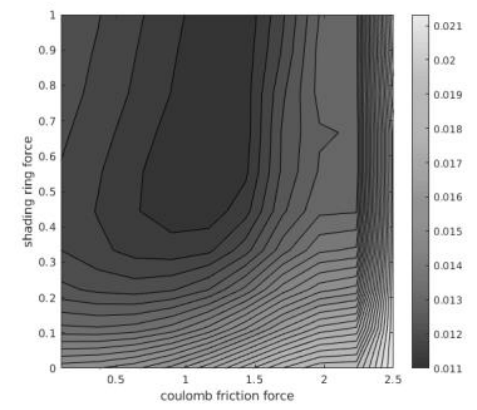

Fig. 9. RMS of current residual with respect to shading ring and dynamic Coulomb friction forces fitting factors - in healthy conditions

\section{B. Identification of the missing forces}

Based on the previous analyses and the two additional forces that were included in the model, their identification was performed. The residual current (see black lines on figure 5) between experiments and simulations was computed every 100 acquisitions per valve and weighted nonlinear least squares were used to estimate the best fitting factors for both forces. Higher weights were used around the important regions highlighted in the previous section. The results for 5 valves show consistent trends and similar life episodes, see figure 9 and figure 5 in the time domain. In addition to the 3 classes considered in the machine learning section, the physical model exhibits a new class that would have been hard to guess without the proposed physical model: when the plunger is not moving as intended but not stucked either (called 'erratic' plunger motion, see figure $5 \mathrm{c}$ ). An essential added value of the physical model with respect to a pure data driven one is the possibility to predict the (unobservable) plunger motion. On figure $5 \mathrm{~b}$, when the plunger motion is large, and the valve leaks one can estimate the plunger displacement $(\sim 0.5 \mathrm{~mm})$.

However, a closer look at figure 5 a shows that the proposed model predicts some plunger motion when it is not supposed to in healthy conditions. This is due to an identifiability issue of the shading ring force. Figure 8 shows that the local minima of the RMS of the residual current are a line. In other words, as soon as the shading ring force fitting factor is higher than $\sim 0.6$, then the current fit is too similar for the optimizer to distinguish values above 0.6, see figure 7. However, the plunger displacement which is not measured and therefore cannot be used for the identification, shows a significant difference from the physical point of view. In healthy conditions, the plunger displacement after the initial hit should be null. Therefore, the shading ring force fitting factor above 0.8 makes more sense from the physical point of view. Unfortunately, the parameters are not totally identifiable by the optimizer in healthy conditions. Nevertheless, the trends presented in figure 9 are quite consistent and give a good physical understanding of the stage of its life at which a given valve is.

\section{CONCLUSIONS AND FUTURE WORK}

The variation of two forces during the lifetime of $\mathrm{AC}$ solenoid valves are found to be sufficient to explain their different stages of degradation. This result allows to perform model-based condition monitoring of the valves. To achieve this, both shading ring and Coulomb forces are included in a classic solenoid valve physical model. The idea to add the shading ring force to the model is derived from feature attribution methods based on a convolutional neural network classifier that highlighted main regions of importance on the current signals.

Future work will work towards the prediction of remaining useful life of AC solenoid valves.

\section{ACKNOWLEDGMENT}

This study was performed in the frameworks of the MODA ICON project. This research was funded by Flanders Make vzw, the strategic research centre for the manufacturing industry, and VLAIO.

\section{REFERENCES}

[1] Perotti, J.M., KEA-71 Smart Current Signature Sensor (SCSS), NASA Tech Report n ${ }^{\circ} 20110000497,2010$

[2] Tsai, H-H., Tseng, C-Y., "Detecting solenoid valve deterioration in inuse electronic diesel fuel injection control systems", Sensors, 2010

[3] Perotti, J. M., Lucena, A., Ihlefeld, C., Burns, B., \& Bassignani, M. (2005). U.S. Patent No. 6,917,203. Washington, DC: U.S. Patent and Trademark Office.

[4] Szente, Viktor, and Janos Vad. "Computational and experimental investigation on solenoid valve dynamics." IEEE/ASME International Conference on Advanced Intelligent Mechatronics, 2001

[5] Taghizadeh, M., A. Ghaffari, and F. Najafi. "Modeling and identification of a solenoid valve for PWM control applications." Comptes Rendus Mecanique, 2009

[6] O. Chapelle and V. N. Vapnik, "Model selection for support vector machines," Advances in Neural Information Processing Systems, S. Solla, T. K. Leen, and K.-R. Muller, Eds. Cambridge, MA: MIT Press, vol. 12, pp. 230-236, 2000.

[7] D. E. Rumelhart, G. E. Hinton, and R. J. Williams, "Learning representation by back propagating errors," Nature, pp. 533-536, 1986.

[8] T. Ince, S. Kiranyaz, L. Eren, M. Askar, and M. Gabbouj, "Real-time motor fault detection by 1-D convolutional neural networks," IEEE Trans. Ind. Electron., vol. 63, no. 11, pp. 7067-7075, 2016.

[9] R. Zhao, R. Yan, Z. Chen, et al. "Deep learning and its applications to machine health monitoring," Mech. Syst. Signal Process., vol. 115, pp. 213-237, 2019.

[10] M. Ancona, E. Ceolini, A.C. Öztireli, and M. Gross, "Towards better understanding of gradient-based attribution methods for deep neural networks," International Conference on Learning Representations (ICLR 2018), 2018. 